\title{
ORIGINAL
}

\section{TENDENCIAS DE LA MORTALIDAD POR ENFERMEDADES CARDIOVASCULARES EN ANDALUCÍA ENTRE 1975 Y 2004}

\author{
Miguel Ruiz-Ramos (1), Teresa Hermosín Bono (2) y Fernando Gamboa Antiñolo (3) \\ (1) Consejería de Salud de la Junta de Andalucía. Sevilla. \\ (2) Centro de Salud de Camas. Servicio Andaluz de Salud. Sevilla. \\ (3) Hospital del Tomillar. Sevilla. Servicio Andaluz de Salud. Sevilla.
}

\section{RESUMEN}

Fundamento: Las enfermedades cardiovasculares están entre las primeras causas de mortalidad en los países industrializados. El objetivo de este trabajo es conocer las tendencias de la mortalidad por enfermedades isquémicas del corazón (EIC) y enfermedades cerebrovasculares (ECV) en Andalucía entre 1975 y 2004.

Método: Con las defunciones por EIC y ECV de las estadísticas oficiales y las correspondientes poblaciones se calcularon las tasas brutas (TB), ajustadas por edad (TS) y Años Potenciales de Vida Pedidos (APVP). Para cuantificar las tendencias y sus puntos de cambio se realizó el análisis con regresión joinpoint.

Resultados: En las mujeres las muertes por EIC pasaron de 2.086 en el año 1975 a 3.336 en el 2004, la TS descendió de 74,29 defunciones por 100.000 mujeres a 50,94, y los APVP pasaron de 173,65 años por 100.000 mujeres a 90,56. En los hombres el número de defunciones pasó de 2.854 defunciones en el 1975 a 4085 en el año 2004, la TS descendió de 147, 67 defunciones por 100.000 hombres a 104,96. Igual comportamiento tuvieron los APVP entre el primer y último año de la serie con unos valores de 716,46 años por 100.000 hombres y 460,04. Para las ECV en las mujeres las defunciones en números absolutos pasaron de 4.712 a 4.221 , la TS pasó de 166,00 defunciones por 100.000 mujeres a 62,08 y los APVP de 338,08 años por 100.000 mujeres a 87,63 . En los hombres el número de defunciones pasó de 3.714 a 2.951, la TS de 206,88 defunciones por 100.000 en 1975 a 76,12 en 2004, y la tasa de APVP de 533,12 años por 100.00 hombres a 182,38.

Conclusiones: La tendencia de la mortalidad por EIC no fue constante ni en las mujeres ni en los hombres aunque en ambos siempre ha sido descendente, siendo el descenso estadísticamente significativo. La disminución de las ECV fue tan importante que tanto los números absolutos como las tasas brutas fueron menores en los últimos años de la serie estudiados que en los primeros, a pesar el envejecimiento de la población andaluza.

Palabras clave: Mortalidad. Enfermedades cardiovasculares. Tendencias. Andalucía.

Correspondencia:

Miguel Ruiz Ramos

Instituto de Estadística de Andalucía.

Pabellón de Nueva Zelanda

Leonardo Da Vinci, s/n

Isla de la Cartuja. 41071. SEVILLA

Correo electrónico: miguel.ruiz.ext@juntadeandalucia.es

\section{ABSTRACT \\ Trends in Mortality Due to Cardiovascular Diseases in Andalusia, Spain (1975-2004)}

Background: Cardiovascular diseases are ranked among the leading causes of death in the industrialized countries. This study is aimed at ascertaining the mortality trends by ischemic heart disease (IHD) and cerebrovascular diseases (CVD) in Andalusia within the 1975-2004 period.

Method: Based on the official IHD and CVD death statistics and the related populations, the gross rates (GR) and age-adjusted rates (TS) and the Potential Years of Life Lost (PYLL) were calculated. To quantify the trends and their change points, a joinpoint regression analysis was made.

Results: The number of IHD deaths for females rose from 2,086 deaths in 1975 to 3,336 in 2004, the TS having dropped from 74.29 to 50.94 deaths $/ 100,000$ females, the PYLL having dropped from 173.65 years to 90.56 years/100,000 females. The number of deaths for males rose from 2,854 deaths in 1975 to 4,085 in 2004, the TS having dropped from 147, 67 to 104.96 deaths $/ 100,000$ males. The PYLL showed a like behaviour from the first to the last year of the series, showing values of 716.46 and 460.04 years / 100,000 males. For the IHD in females, the number of deaths in absolute numbers dropped from 4,712 to 4,221 , the TS having dropped from 166.00 to 62.08 deaths in females, and the PYLL from 338.08 to 87.63 years / 100,000 females. For males, the number of deaths dropped from 3,714 to 2,951, the TS from 206.88 deaths $/ 100,000$ males in 1975 to $76.12 / 100,000$ males in 2004, and the PYLL dropping from 533.12 to 182.38 years / 100,000 males.

Conclusions: The trend in mortality due to IHD was not constant either among females or males, although it has always been a downward trend, the drop being statistically significant. The drop in the CVD has been such a major one that both the absolute numbers and the gross rates are lower for the most recent years that the first years in the series studied despite the aging of Andalusia's population.

Key word: Mortality. Cardiovascular diseases. Trends. Andalusia. 


\section{INTRODUCCIÓN}

Las enfermedades cardiovasculares fueron la primera causa de muerte en España en el año 2004, representando el $33,3 \%$ del total de defunciones, es decir, una de cada tres. Dentro de este grupo las enfermedades isquémicas del corazón (EIC) fueron la primera causa de muerte entre los hombres con 21.898 defunciones y las enfermedades cerebrovasculares (ECV) entre las mujeres con 20.049 defunciones ${ }^{1}$. La estimación de las incidencias de la EIC estaban en entorno a 100.000 anuales, teniendo en cuenta que según la cohorte de Framingham ${ }^{2}$ un $30 \%$ de estas enfermedades que ocurren en la comunidad tienen un curso clínico silente, es decir, son detectadas tras la realización de un electrocardiograma por los servicios sanitarios en ausencia de síntomas clínicos de enfermedad. Alrededor de un $57 \%$ de los casos incidentes fallecen en los primeros 28 días después de la aparición de la enfermedad, sobre todo debido a la alta letalidad en las personas mayores de 74 años. Se estima que en Andalucía en el año 2002 se produjo un total de 10.294 casos de EIC de los cuales 5.795 murieron en los primeros 28 días ${ }^{3}$.

En el panorama internacional, tanto en morbilidad como en mortalidad España presenta unos de los niveles más bajos, sin embargo la exposición a los factores de riesgo conocidos no es menor en España que en otros países ${ }^{4,5}$. Este fenómeno, conocido como la paradoja francesa, está presente en otras países del mediterráneo, por lo que se ha propuesto la posible existencia de factores protectores en la dieta mediterránea ${ }^{6-8}$. Todavía existe poca información del efecto de los factores de riesgo cardiovascular con bajas tasas de incidencia y $\operatorname{mortalidad}^{9,10}$, aunque actualmente hay datos que sugieren que el efecto de los principales factores de riesgo cardiovascular es similar en todas las poblaciones del mundo. Lo que cambia es el riesgo basal, la incidencia de la enfermedad en la población, y la prevalencia de factores de riesgo entre poblaciones. Y esto fundamentaría que el efecto de los principales factores de riesgo cardiovascular en poblaciones de baja incidencia pueda ser diferente al de las poblaciones con altos niveles de mortalidad $^{11}$.

Por otro lado, la información disponible en España y en Europa es en general dispersa y poco comparable ${ }^{12}$, por lo cual toman especial relevancia los datos de mortalidad procedentes de las estadísticas oficiales de mortalidad que, aún con sus limitaciones, son la principal fuente de información de toda la población, comparables y de fácil acceso.

En Andalucía y en España contar con un sistema sanitario de atención primaria con cobertura universal puede ser una buena alternativa a los registros de base poblacional dirigidos a obtener información epidemiológica de este importante problema de salud. Los médicos de familia están cualificados y capacitados para detectar en los pacientes factores de riesgo cardiovascular, por ser éstos fácilmente identificables y, por lo tanto, está información es recogida de manera rutinaria en la práctica clínica de atención primaria $^{13}$.

Andalucía es una de las zonas de España con mayores tasas de mortalidad por enfermedades cardiovasculares, tanto en los hombres como en las mujeres, sobre todo en las provincias de Cádiz, Huelva y Sevilla ${ }^{14,15}$. Se han realizado pocos estudios que aclaren estas circunstancias. El objetivo de este trabajo es describir la mortalidad por las enfermedades isquémicas del corazón y las enfermedades cerebrovasculares en Andalucía desde el año 1975 hasta el 2004, para hombres y mujeres por separado. 


\section{MATERIAL Y MÉTODOS}

Las defunciones por enfermedades cardiovasculares por edad y sexo corresponden a las publicadas por el Instituto Nacional de Estadística durante los años de 1975 al 1991 y por el Instituto de Estadística de Andalucía para el periodo 1992-2004. En los años que se incluyen se han producido cambios en las revisiones de la Clasificación Internacional de Enfermedades, de 1975 a 1979 la octava, de 1980 a 1998 la novena y desde 1999 al 2004 la décima. Para el cálculo de indicadores se han utilizado las poblaciones proyectadas por el INE desde 1971 a 2026.

De todas las enfermedades cardiovasculares se han estudiado las EIC que corresponden a los códigos del 410 al 414 de la CIE-8 y CIE-9 y al I20-I25 de la CIE-10, y ECV con los códigos de 430-438 de la CIE8 y CIE-9 y la I60-I69 de la CIE-10.

Con estos datos se calcularon las tasas brutas y ajustadas por edad, dividiendo el número de defunciones ocurridas en un año determinado por la población en riesgo a mitad de periodo. Para calcular las tasas ajustadas por edad, útiles para la comparación entre poblaciones u una misma población a lo largo del tiempo, se utilizaron la población estándar europea. Para estimar los cambios de tendencia observada a lo largo del tiempo se construyeron modelos segmentados de regresión de Poisson, conocido también como análisis con regresión joinpoint. Su resultado es doble: identifican el momento en que se producen los cambios significativos de la tendencia y estiman la magnitud del aumento o el descenso observado en cada intervalo a través de los porcentajes de cambio anual (PCA). En los modelos utilizados se establecieron hasta un máximo de dos puntos de cambio (joinpoint) y la significación estadística se fijó en el $0,05 \%{ }^{16}$.

\section{RESULTADOS}

En las mujeres de Andalucía las muertes por enfermedades isquémica del corazón pasaron de 2.086 en el año 1975 a 3.336 en el 2004, la tasa bruta de mortalidad ascendió de 66,91 defunciones por 100.000 mujeres en 1975 a 88,82 en 2004, sin embargo la tasa ajustada por edad descendió de 74,29 defunciones por 100.000 mujeres a 50,94 entre 1975 y 2004, y la tasa ajustada por edad de Años Potenciales de Vida Perdidos paso de 173,65 años por 100.000 mujeres en 1975 a 90,56 en el 2004 (tabla 1).

En los hombres el número de defunciones por EIC pasó de 2.854 defunciones en el 1975 a 4.085 en el año 2004, la TB pasó de 94,96 defunciones por 100.000 hombres a 112,54 en el mismo periodo, la TS descendió de 147,67 defunciones por 100.000 hombres a 104,96 entre 1975 y 2004, e igual comportamiento tuvieron los APVP entre el primer y último año de la serie con unos valores de 716,46 años por 100.000 hombres y 460,04.

La cuantificación de las tendencias de la mortalidad por enfermedades isquémicas del corazón se muestra en la tabla 3 y en la figura 1. La tendencia no fue homogénea ni en las mujeres ni en los hombres. En las primeras entre 1975 y 1987 se produjo un PCA de $-1,96 \%$ estadísticamente significativo, para el periodo de 1987 a 1998 cambió a una tendencia ascendente aunque no significativa estadísticamente, para luego descender de una manera más intensa desde 1998 hasta el 2004 con un PAC de $-3,21 \%$ estadísticamente significativo. En los hombres la tendencia fue muy parecida (tabla 3), con un periodo entre 1975 y 1989 descendente con un PCA de $-1,23 \%$ estadísticamente significativo, para producirse un ligero ascenso en el periodo entre 1989 y 1998 con PCA del 0,20\%, y un último periodo entre 1998 
Tabla 1

Mortalidad por enfermedades isquémicas del corazón en Andalucía entre 1975 y 2004

\begin{tabular}{|c|c|c|c|c|c|c|c|c|}
\hline \multirow{2}{*}{ Año } & \multicolumn{4}{|c|}{ Mujeres } & \multicolumn{4}{|c|}{ Hombres } \\
\hline & Defun & TB & TS & APVP & Defun & TB & TS & APVP \\
\hline 1975 & 2086 & 66,91 & 74,29 & 173,65 & 2854 & 94,96 & 147,67 & 716,76 \\
\hline 1976 & 2238 & 71,39 & 77,36 & 161,48 & 2916 & 96,42 & 146,92 & 713,33 \\
\hline 1977 & 2024 & 64,13 & 68,74 & 148,96 & 2774 & 91,03 & 137,92 & 686,80 \\
\hline 1978 & 2127 & 66,86 & 69,26 & 140,92 & 2893 & 94,13 & 139,33 & 722,50 \\
\hline 1979 & 2331 & 72,67 & 74,39 & 170,01 & 2925 & 94,30 & 137,20 & 695,14 \\
\hline 1980 & 1924 & 59,47 & 59,78 & 156,04 & 2827 & 90,26 & 128,04 & 758,62 \\
\hline 1981 & 2125 & 65,11 & 64,06 & 162,06 & 2983 & 94,22 & 131,94 & 716,88 \\
\hline 1982 & 2272 & 68,91 & 65,44 & 166,69 & 3137 & 97,97 & 135,07 & 715,29 \\
\hline 1983 & 2302 & 68,98 & 64,45 & 160,82 & 3042 & 93,75 & 127,95 & 646,84 \\
\hline 1984 & 2357 & 69,74 & 64,56 & 155,83 & 3169 & 96,35 & 129,96 & 693,05 \\
\hline 1985 & 2357 & 68,66 & 62,49 & 133,87 & 3286 & 98,76 & 131,73 & 690,44 \\
\hline 1986 & 2287 & 66,35 & 58,30 & 140,96 & 3258 & 97,11 & 126,25 & 664,46 \\
\hline 1987 & 2328 & 57,11 & 57,93 & 139,81 & 3245 & 96,14 & 122,97 & 670,00 \\
\hline 1988 & 2486 & 71,39 & 59,76 & 129,89 & 3383 & 99,90 & 124,33 & 642,76 \\
\hline 1989 & 2466 & 70,62 & 58,03 & 128,08 & 3338 & 98,39 & 121,42 & 606,48 \\
\hline 1990 & 2596 & 74,09 & 59,73 & 138,95 & 3359 & 98,75 & 119,88 & 590,34 \\
\hline 1991 & 2735 & 77,64 & 61,60 & 141,55 & 3578 & 104,68 & 123,95 & 619,68 \\
\hline 1992 & 2791 & 78,66 & 60,82 & 116,09 & 3658 & 106,28 & 125,25 & 633,40 \\
\hline 1993 & 2915 & 81,35 & 61,07 & 114,78 & 3777 & 108,72 & 125,53 & 576,45 \\
\hline 1994 & 2894 & 79,93 & 59,69 & 115,84 & 3530 & 100,58 & 115,03 & 529,47 \\
\hline 1995 & 3066 & 84,00 & 61,29 & 114,28 & 3866 & 109,23 & 122,60 & 569,09 \\
\hline 1996 & 3155 & 85,86 & 60,77 & 113,07 & 3981 & 111,86 & 123,65 & 561,98 \\
\hline 1997 & 3263 & 88,85 & 61,21 & 126,31 & 3951 & 111,13 & 121,07 & 530,83 \\
\hline 1998 & 3446 & 93,70 & 62,16 & 105,35 & 4165 & 117,03 & 125,92 & 569,46 \\
\hline 1999 & 3388 & 91,83 & 59,62 & 108,82 & 4178 & 117,03 & 122,23 & 500,85 \\
\hline 2000 & 3316 & 89,59 & 56,79 & 95,51 & 4134 & 115,44 & 118,56 & 543,13 \\
\hline 2001 & 3122 & 84,06 & 52,52 & 106,25 & 4024 & 112,01 & 112,36 & 512,13 \\
\hline 2002 & 3328 & 89,28 & 54,04 & 100,32 & 4075 & 113,05 & 111,40 & 520,94 \\
\hline 2003 & 3395 & 90,74 & 53,82 & 108,95 & 4297 & 118,80 & 114,14 & 525,63 \\
\hline 2004 & 3336 & 86,82 & 50,94 & 90,58 & 4085 & 112,54 & 104,96 & 460,04 \\
\hline
\end{tabular}

Defun, número de defunciones; TB, tasa bruta de mortalidad; TS, tasas ajustada por edad de mortalidad. APVP, tasa ajustada por edad de Años Potenciales de Vida Perdidos.

y 2004 durante el cual volvió a ser descendente de una manera significativa con un PCA de $-2,35 \%$. Tanto en los hombres como en las mujeres la tendencia para el conjunto del periodo, desde 1975 al 2004 fue descendente con PCA estadísticamente significativos. En las mujeres el PCA fue de $-0,94 \%$ y en los hombres de $0,79 \%$.
En la tabla 2 se presentan los resultado de la mortalidad por enfermedades cerebrovasculares en Andalucía en el periodo de 1975 a 2004 para mujeres y hombres. En las mujeres las defunciones en números absolutos pasaron de 4.712 en el año 1975 a 4.221 en el 2004, la TB descendió de 151,14 a 112, 39 defunciones por 100.000 mujeres, la TS pasó de 166,00 a 62,08 
Tabla 2

Mortalidad por enfermedades cerebrovasculares en Andalucía entre 1975 y 2004

\begin{tabular}{|c|c|c|c|c|c|c|c|c|}
\hline \multirow{2}{*}{ Año } & \multicolumn{4}{|c|}{ Mujeres } & \multicolumn{4}{|c|}{ Hombres } \\
\hline & Defun & TB & TS & APVP & Defun & TB & TS & APVP \\
\hline 1975 & 4712 & 151,14 & 166,00 & 338,08 & 3714 & 123,58 & 206,88 & 533,12 \\
\hline 1976 & 4951 & 157,93 & 169,71 & 335,53 & 3800 & 125,65 & 203,25 & 536,07 \\
\hline 1977 & 4749 & 150,46 & 160,52 & 329,09 & 3578 & 117,42 & 191,62 & 487,86 \\
\hline 1978 & 4829 & 151,80 & 158,75 & 305,44 & 3715 & 120,87 & 191,55 & 519,40 \\
\hline 1979 & 4954 & 154,44 & 157,99 & 296,97 & 3682 & 116,70 & 186,69 & 496,76 \\
\hline 1980 & 5006 & 154,74 & 154,58 & 274,63 & 3552 & 113,41 & 176,64 & 410,96 \\
\hline 1981 & 5162 & 158,16 & 153,63 & 279,81 & 3674 & 116,05 & 175,11 & 471,92 \\
\hline 1982 & 4761 & 144,41 & 136,35 & 238,87 & 3302 & 103,13 & 152,31 & 393,14 \\
\hline 1983 & 5097 & 152,74 & 140,18 & 234,35 & 3424 & 105,52 & 155,01 & 349,91 \\
\hline 1984 & 5134 & 151,91 & 138,33 & 232,94 & 3587 & 109,06 & 157,79 & 447,13 \\
\hline 1985 & 5045 & 147,59 & 133,41 & 228,96 & 3615 & 108,65 & 156,33 & 384,80 \\
\hline 1986 & 4791 & 136,99 & 119,82 & 216,41 & 3246 & 96,81 & 136,22 & 361,78 \\
\hline 1987 & 4882 & 140,74 & 119,60 & 234,56 & 3310 & 98,34 & 133,13 & 374,10 \\
\hline 1988 & 4792 & 137,61 & 112,26 & 186,12 & 3364 & 99,34 & 131,85 & 332,66 \\
\hline 1989 & 4546 & 130,19 & 103,87 & 183,53 & 3221 & 94,94 & 123,72 & 317,85 \\
\hline 1990 & 4429 & 126,41 & 98,06 & 163,42 & 3075 & 90,40 & 116,88 & 284,43 \\
\hline 1991 & 4660 & 132,29 & 100,39 & 163,48 & 3191 & 93,36 & 117,13 & 292,15 \\
\hline 1992 & 4485 & 126,41 & 94,41 & 145,07 & 2956 & 85,89 & 106,88 & 289,31 \\
\hline 1993 & 4478 & 124,97 & 91,04 & 122,80 & 3032 & 87,27 & 106,08 & 263,98 \\
\hline 1994 & 4379 & 120,95 & 86,95 & 128,21 & 2795 & 79,64 & 95,40 & 215,66 \\
\hline 1995 & 4278 & 117,13 & 83,05 & 128,84 & 2910 & 82,22 & 96,23 & 250,63 \\
\hline 1996 & 4239 & 115,36 & 79,12 & 112,38 & 2961 & 83,20 & 95,66 & 245,28 \\
\hline 1997 & 4174 & 113,66 & 75,49 & 113,35 & 2761 & 77,66 & 87,61 & 207,45 \\
\hline 1998 & 4190 & 113,94 & 72,96 & 83,09 & 2893 & 81,29 & 91,01 & 202,46 \\
\hline 1999 & 4483 & 121,51 & 78,55 & 107,68 & 3206 & 89,86 & 97,85 & 238,51 \\
\hline 2000 & 4196 & 113,36 & 69,26 & 90,86 & 2860 & 79,86 & 84,56 & 189,03 \\
\hline 2001 & 4310 & 116,04 & 69,61 & 104,36 & 2942 & 81,89 & 83,74 & 208,28 \\
\hline 2002 & 4288 & 115,04 & 67,50 & 111,53 & 3007 & 83,42 & 83,16 & 208,07 \\
\hline 2003 & 4606 & 123,11 & 69,90 & 106,15 & 3212 & 88,80 & 86,21 & 182,19 \\
\hline 2004 & 4221 & 112,39 & 62,08 & 87,63 & 2951 & 81,30 & 76,12 & 182,38 \\
\hline
\end{tabular}

Defun, número de defunciones; TB, tasa bruta de mortalidad; TS, tasas ajustada por edad de mortalidad. APVP, tasa ajustada por edad de Años Potenciales de Vida Perdidos.

defunciones por 100.000 mujeres y los APVP de 338,08 a 87,63 años por 100.000 mujeres. En los hombres el número de defunciones pasó de 3.714 a 2.951, la TB de 123,58 a 81,30 defunciones por 100.000 hombres, la TS de 206,88 a 76,12 defunciones por 100.000 en, y la tasa de APVP de 533,12 años a 182,38 por 100.00 hombres.
La tendencia de la mortalidad por enfermedades cerebrovasculares no fue constante ni en las mujeres ni en los hombres aunque en ambos siempre fue descendente y si se considera el conjunto de años estudiado fue fuertemente descendente como se puede apreciar en la tabla 3 y en la figura 2, un PCA entre 1975 y 2004 de $-3,60 \%$ en las mujeres y de $-3,52 \%$ en los hom- 
Tabla 3

Tendencia de la mortalidad por enfermedades cardiovasculares en Andalucía entre 1975 y 2004

\begin{tabular}{|l|c|c|c|c|c|c|c|c|}
\hline & PCA Global & Periodo 1 & PCA & Periodo 2 & PCA & Periodo 3 & PCA \\
\hline Isquémicas & $-0,94^{*}$ & $1975-1987$ & $-1,96^{*}$ & $1987-1998$ & 0,40 & $1998-2004$ & $-3,21^{*}$ \\
\hline Mujeres & $-0,79 *$ & $1975-1989$ & $-1,23^{*}$ & $1989-1998$ & 0,20 & $1998-2004$ & $-2,35^{*}$ \\
\hline Hombres & $-3,60^{*}$ & $1975-1981$ & $-1,69^{*}$ & $1981-1997$ & $-4,23^{*}$ & $1997-2004$ & $-2,21^{*}$ \\
\hline Cerebrovasculares & $-3,52^{*}$ & $1975-1995$ & $-3,83^{*}$ & $1995-2004$ & $-2,21^{*}$ & \\
\hline Mujeres &
\end{tabular}

PCA global, porcentaje de cambio anual entre 1975 y 2004; PCA, porcentaje de cambio anual, *estadísticamente significativo, p<0,05.

Figura 1

Mortalidad por cardiopatía isquémica en Andalucía entre 1975 y 2004
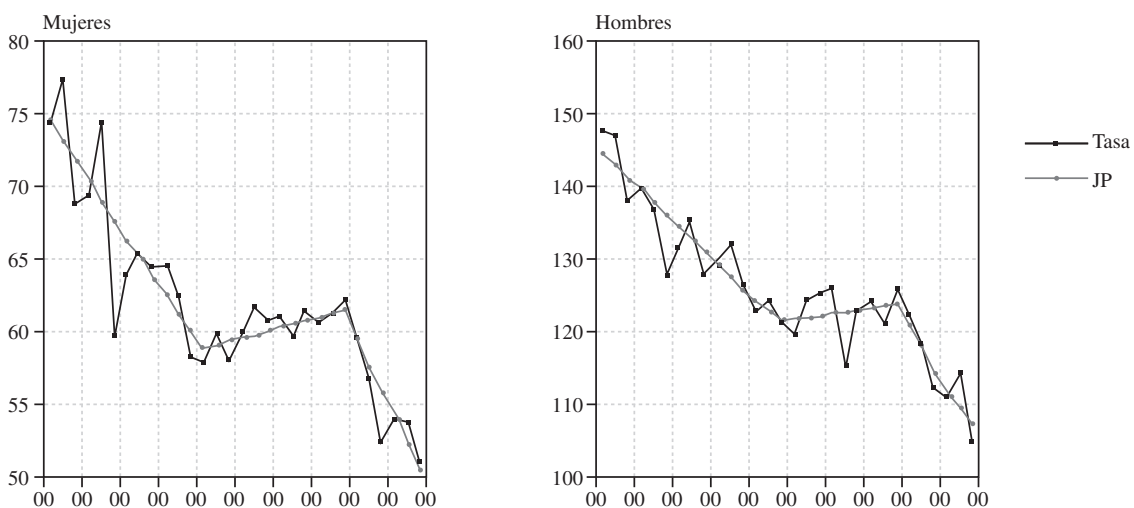

Figura 2

Mortalidad por accidentes cerebrovasculares en Andalucía entre 1975 y 2004
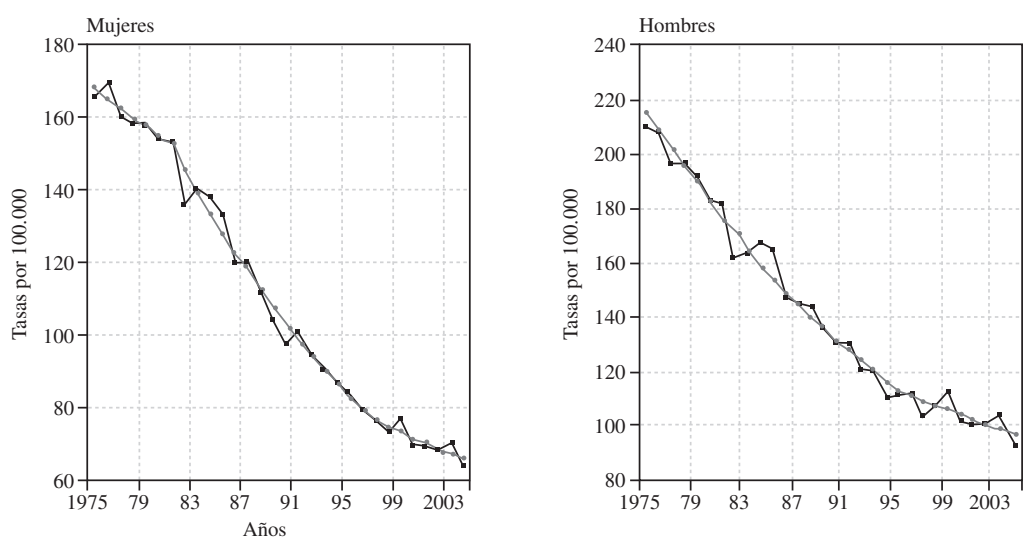

$\longrightarrow$ Tasa 
bres. En las mujeres se detectaron dos puntos de cambio estadísticamente significativos que conformaron tres periodos: el primero de 1975 a 1981 con un PCA de $-1,69 \%$ que, aunque estadísticamente significativo, fue menos intenso que el del periodo entre 1981 y 1997 con un PCA de $-4,23 \%$ y el de 1997 al 2004 con un PCA de $-2,21 \%$. En los hombres se detectó un punto de cambio estadísticamente significativo en torno al año 1995, que determinó dos periodos, el primero entre 1975 y 1995 con un PCA de $-3,38$ y el segundo entre 1995 y 2004 con -2,21, ambos estadísticamente significativos.

\section{DISCUSIÓN}

Las enfermedades isquémicas del corazón y las enfermedades cerebrovasculares en Andalucía desde 1975 al 2004 han tenido un importante descenso tanto en las mujeres como en los hombres. En especial hay que destacar el descenso de las enfermedades cerebrovasculares, tan importante que tanto los indicadores crudos como los números absolutos y las tasas brutas de mortalidad son menores en los últimos años de la serie estudiados que en los primeros. La enfermedad isquémica del corazón ha tenido un comportamiento diferente, los indicadores brutos presentan valores más elevados en los últimos años de la serie que en los primeros. Cuando se ajustan por edad mediante la estandarización directa pasan a ser descendentes, pudiéndose atribuir el aumento del número de defunciones y las tasas brutas al efecto del envejecimiento de la población andaluza en los treinta años estudiados. El descenso no ha sido constante, sino que ha habido un periodo en torno a finales de los años ochenta hasta el año 1998 en que la EIC presentaba un tendencia ascendente, que podrían hacer pensar en un cambio de tendencia o en una estabilización de la mortalidad por esta causa tanto en los hombres como en las mujeres. Sin embargo, es a partir de los año 1998 cuando se produjo el cambio de tendencia claramente descendente. Éste coincide con la introducción de la décima revisión de la CIE, en el año 1999 en España. Este hecho se podría atribuir a que se ha producido un artefacto en la clasificación de los diagnósticos de causa de muerte de estas causas, con los consiguientes cambios de clasificación. Sin embargo si esto hubiese ocurrido en los años siguientes no habría seguido descendiendo, ya que a partir de 1999 se clasificaron con los mismos criterios todos los años. Además los estudios realizados en España de comparación entre la novena y décima revisión de la CIE concluyen que la CIE-10 no ha introducido grandes cambios en la clasificación de la enfermedad isquémica del corazón ${ }^{17,18}$. Por otro lado, este descenso no es exclusivo de Andalucía sino que también se ha puesto de manifiesto en otros trabajos realizados en el conjunto de España ${ }^{19}$.

Descartado que el descenso de la mortalidad por estas causas sea debido al cambio de versión de la clasificación, habrá que buscar otras causas que lo expliquen. Cambios en los factores de riesgo cardiovascular, diferencias en el nivel socioeconómico y el acceso a los servicios de salud pueden ser clave para conocer lo que ha ocurrido en la población andaluza durante el periodo estudiado. El aumento de consumo de frutas y el descenso en la ingesta de vino podrían estar relacionados con el descenso de la mortalidad de ECV y EIC en España ${ }^{20}$ y en otros países ${ }^{21}$. Pero quizás la causa más importante sea el aumento de la supervivencia por una mejora en las terapias de estas enfermedades ${ }^{22,23}$. En otros países se han realizado estudios sobre las causas del descenso de la mortalidad por enfermedades isquémicas del corazón atribuyendo la mitad de este descenso a la reducción de los factores de riego y la otra mitad a las terapias médicas basadas en evidencias ${ }^{24}$, lo mismo ha ocurrido con el descenso de la incidencia y mortalidad de ECV en el Reino 
Unido atribuible a un mejor tratamiento preventivo y de los factores de riesgo de la enfermedad $^{25}$

Íntimamente relacionados con los resultados de este estudio, al tiempo que son sus principales limitaciones, son la fiabilidad de los datos sobre mortalidad por enfermedades cardiovasculares en general y más específicamente por EIC y ECV que, a su vez, por la magnitud que representan pueden tener un efecto importante en la mortalidad por otras causas. En Andalucía no existen estudios que valoren la fiabilidad de las estadísticas de causas de muerte, y el conocimiento sobre este importante tema se refiere a estudios realizados hace años, en una zona determinada de España ${ }^{26}$. Un problema añadido a la calidad de esta información es que el mayor número de defunciones se produce en personas muy mayores en las que discernir cuál ha sido la causa que ha producido la muerte puede ser difícil por presentar pluripatología y con frecuencia no estar realizada la certificación de causa de muerte su medico de cabecera, con lo cual la calidad del certificado de defunción puede ser bastante deficiente. Esto ocurre, sobre todo cuando la muerte tiene lugar fuera del medio hospitalario e intervienen los médicos de las compañías funerarias.

Los modelos de regresión joinpoint se han utilizado para estimar los porcentajes anuales tanto de las EIC como de las ECV. Esta técnica estadística describe los cambios de tendencias de cada uno de los sucesivos periodos y los aumentos o descensos dentro de los mismos después de identificar el mejor ajuste del modelo. También identifica los años (puntos) en los que se han producido un cambio significativo de las tendencias. Sin embargo tiene algunas limitaciones que hay que conocer para un uso correcto. En el cálculo de los PCA el método asume que los cambios son constantes en el intervalo de tiempo y esto puede no ser cierto. Por lo tanto puede haber problema para comparar con otros trabajos realizados con los mismos datos pero en periodos diferentes ${ }^{27}$.

A pesar de las limitaciones expuestas con anterioridad, parece claro que aún con el descenso de las enfermedades cardiovasculares en Andalucía, este problema cada vez va a representar una mayor magnitud tanto como causa de muerte como de morbilidad, a causa del envejecimiento de la población andaluza en los próximos años. Lo que convendría tener en cuenta para disponer de los recursos adecuados para una correcta atención del problema.

\section{BIBLIOGRAFÍA}

1. Instituto Nacional de Estadística. Defunciones según la causa de muerte 2004 (citado 2 de Mar 2007). Disponible en: http://www.ine.es/prensa/np410.pdf.

2. Kannel WB. Silent myocardial ischemia and infarction: insights from Framingham Study. Cardiol Clin 1986; 4: 583-91.

3. Marrugat J, Elosua R, Martí H. Espidemiology of Ischaemic Heart Diseases in Spain: Estimation of the Number of Cases and Trends from 1997 to 2005. Rev Esp Cardiol 2002; 55(4): 337-46.

4. Medrano MJ, Cerrato E, Boix R, Delgado M. Prevalencia de factores de riesgo cardiovascular en España: metaanálisis de estudios transversales. Med Clin (Barc). 2005;124:606-12.

5. Masia R, Pena A, Marrugat J, Sala J, Vila J, Pavesi M, Covas M, Aubo C, Elosua R, and the REGICOR investigators. High prevalence of cardiovascular risk factors in Gerona, Spain, a province with low myocardial infarction incidence. J Epidemiol Comm Health. 1998;52:707-15.

6. Ferrieres J. The French paradox: lessons for other countries. Heart. 2004;90:107-111.

7. Fernandez-Jarne E, Martinez-Losa E, Prado-Santamaria M, Brugarolas-Brufau C, Serrano-Martinez M, Martinez-Gonzalez MA. Risk of first nonfatal myocardial infarction negatively associated with olive oil consumption: a case-control study in Spain. Int J Epidemiol. 002;31:474-80.

8. Wollin S, Jones P. Alcohol, red wine and cardiovascular disease. J Nutr. 2001;131:1401-4. 
9. Fraser GE, Straham TM, Sabaté J, Beeson WL, Kissinger D. Effects of traditional coronary risk factors on rates of incident coronary events in a low-risk population. The Adventist Health Study. Circulation. 1992;86:406-413.

10. Conroy RM, Pyorala K, Fitzgerald AP, Sans S, Menotti A, De Backer G, De Bacquer D, Ducimetiere P, Jousilahti P, Keil U, Njolstad I, Oganov RG, Thomsen T, Tunstall-Pedoe H, Tverdal A, Wedel H, Whincup P, Wilhelmsen L, Graham IM, SCORE project group. Estimation of ten-year risk of fatal cardiovascular disease in Europe: the SCORE project. Eur Heart J. 2003;24:987-1003.

11. Marrugat J, D'Agostino R, Sullivan L, Elosua R, Wilson P, Ordovas J, Solanas P, Cordon F, Ramos R, Sala J, Masia R, Kannel WB. An adaptation of the Framingham coronary heart disease risk function to European Mediterranean areas. J Epidemiol Community Health. 2003;57:634-8. doi: 10.1136 .

12. The EUROCISS Working Group. Coronary and cerebrovascular population-based registers in Europe: are morbidity indicators comparable? Results from the EUROCISS project. Eur J Public Health. 2003;13:55-60.

13. Marín A, Medrano MJ, González J et al. Risk of ischaemic heart disease and acute myocardial infarction in a Spanish population: observational prospective study in a primary-care setting. BMC Public Health. 2006; 6: 38.

14. Olalla MT, Medrano MJ, Sierra MJ, Almazan J. Cerebrovascular mortality in Spain. Rev Neurol. 1999 Nov 1-15;29(9):872-8.

15. Boix Martinez R, Aragones Sanz N, Medrano Albero MJ. Trends in mortality from ischemic heart disease in 50 Spanish provinces. Rev Esp Cardiol. 2003 Sep;56(9):848-9.

16. Kim HJ, Fay MP, Feuer EJ, Midthune DN. Permutation test for joinpoint regression with applications to cancer rates. Statist Med. 2000; 19: 335351.

17. Cirera Suarez L, Rodriguez Barranco M, Madrigal de Torres E, et al. Correspondences from 10th to 9th Revision of the International Classification of Diseases in the causes of death lists of the National Institute of Statistics and the Regional Health
Authority of Murcia in Spain. Rev Esp Salud Publica. 2006 Mar-Apr;80(2):157-75.

18. Cano-Serral G, Perez G, Borrell C, COMPARA Group. Links Comparability between ICD-9 and ICD-10 for the leading causes of death in Spain. Rev Epidemiol Sante Publique. 2006 Sep; 54(4): 355-65.

19. Boix Martinez R, Aragones Sanz N, Medrano Albero MJ. Trends in mortality from ischemic heart disease in 50 Spanish provinces. Rev Esp Cardiol. 2003 Sep;56(9):850-6.

20. Rodriguez Artalejo F, Guallar-Castillon P, Banegas Banegas JR, et Al. Consumption of fruit and wine and the decline in cerebrovascular disease mortality in Spain (1975-1993). Stroke. 1998 Aug;29(8):1556-61.

21. Ness AR, Powles JW. Fruit and vegetables, and cardiovascular disease: a review. Int J Epidemiol. 1997 Feb;26(1):1-13.

22. Sala J, Marrugat J, Masiá R, Porta-Serra M, and REGICOR investigators. Improvement in survival after myocardial infarction between 1978-85 and 1986-88 in REGICOR study. Eur Heart J. 1995; $52: 767-75$.

23. Gil M, Marrugat J. Sala J et Al. Relationship of therapeutic improvement and 28 day case fatality in patients hospitalized with acute myocardial infarction between 1978 and 1993 in the REGICOR study. Circulation. 1999; 99:1767-73.

24. Ford ES, Ajani UA, Croft JB, et al . Explaining the decrease in U.S. deaths from coronary disease, 1980-200. N Engl J Med. 2007; 356: 2388-2398

25. Rothwell PM, Coull AJ, Giles MF et al. Change in stroke incidence, mortality, case-fatality, severity, and risk factors in Oxforshire, UK from 1981 to 2004 (Oxford Vascular Study). Lancet. 2004; 363:1925-33.

26. Benavides FG, Bolúmar F, Peris R. Quality of Death Certificate in Spain. Am J Public Health. 1989;79(10):1352-4.

27. Howe HL, Wingo P, Thun MJ et al. Annual report to the nation on the status of cancer (1973 through 1998), featuring cancer with recent increasing trends. J Natl Cancer Inst. 2001; 93(11):824-42. 
